\title{
TEORÍAS PARA LA PAZ Y PERSPECTIVAS AMBIENTALES DEL DESARROLLO COMO DIÁLOGOS DE IMPERFECTOS
}

\author{
MARIO HERNÁN LÓPEZ BECERRA ${ }^{1}$ \\ mariohl2006@yahoo.es.
}

Manizales, 2011-04-18 (Rev. 2011-08-29)

Resumen:

Desde finales de la segunda guerra mundial, en distintos centros e institutos de Investigación, se están desarrollando teorías sobre y para la paz (quizá sea más adecuado referirse a las paces, dada la inobjetable polisemia del concepto) que la vinculan a la cesación de las violencias o, de manera más amplia, a la solución pacífica de los conflictos. Una de los desarrollos más recientes es la Paz Imperfecta, a través de ella es posible detectar acciones que crean paz a pesar de estar en contextos de alta conflictividad y violencia. En esta línea de análisis la paz como ideal absoluto y perpetuo es sustituida por una gama amplia y variopinta de paces posibles, abriendo, de esta manera, un espacio sugerente para examinar las probables conexiones entre los pensamientos y acciones de pacifistas y ambientalistas, teniendo como fondo un juicio crítico común al modelo de desarrollo imperante.

El presente texto hace un recorrido teórico por algunas de las reflexiones más estimulantes sobre la paz; en la ruta, el documento construye conexiones entre una de estas perspectivas (paz imperfecta) y algunas de las variantes del pensamiento ambiental; como se sabe, estas últimas han dado paso a múltiples visiones del desarrollo alternativo, buena parte de ellas centradas en la búsqueda de la sustentabilidad.

\section{Palabras Clave:}

Paz Negativa, Paz Positiva, Paz imperfecta, Violencia directa, Violencia indirecta, Violencia Cultural, Violencia estructural, Conflicto, Perspectiva Ambiental, Desarrollo Sustentable.

\section{THEORIES FOR PEACE AND DEVELOPMENT ENVIRONMENTAL PERSPECTIVES AS IMPERFECT DIALOGUES}

\begin{abstract}
:
Since the end of the Second World War, theories about and for peace (probably it would be more adequate to refer to them as peaces because of the unobjectionable polysemy of this concept) which link it to the ceasing of violence or, in a wider sense, to pacific problem solving, have been developed. One of the most recent developments is the so called Imperfect Peace, through which it is possible to detect actions that generate peace in spite of being in high conflictive and violent contexts. In this line of analysis, peace as an absolute and perpetual ideal is substituted by a wide and varied spectrum of possible peaces, opening in this way suggestive space to examine the possible connection between pacifists and environmentalists' thoughts and actions, having as a background a critical judgment common to the prevailing development model.
\end{abstract}

This article makes a theoretical scan on some of the most stimulant reflections about peace; on the road the document builds connections between one of these perspectives (Imperfect Peace) and some of the 
environmental thought variables; as it is well known these last ones have given way to multiple visions of alternative development, many of then focused on the search for sustainability.

\section{Keywords:}

Negative Peace, Positive Peace, Imperfect Peace, direct violence, indirect violence, cultural violence, structural violence, conflict, environmental perspective, sustainable development.

\section{Consideraciones Iniciales}

En razón a la amplitud y complejidad que revisten los temas comprometidos, los alcances del presente texto están circunscritos a la revisión de algunas teorías, conceptos y debates sobre la paz -o más exactamente sobre las paces en un sentido necesariamente polisémico dada la complejidad histórica, teórica, axiológica y práctica que implica cualquier definición- planteados por algunos autores durante el siglo XX y los inicios del XXI. Una parte de la literatura que aquí se referencia está asociada con una tradición investigativa que liga las reflexiones sobre la paz a los estudios sobre la violencia.

En años recientes, se ha venido avanzando hacia nuevos abordajes investigativos a partir de los cuales se adoptan e incorporan elementos de diferentes enfoques metodológicos, con marcos inter y transdisciplinarios y con fundamento en valores pacifistas; estos abordajes invitan insistentemente a explorar en las relaciones entre los pensamientos pacifistas y ambientalistas.

De forma general, se pueden identificar dos tendencias en los estudios actuales de y para la paz: de un lado se encuentran aquellos que relacionan la paz con la violencia y sus distintas modalidades, y, de otro, los que relacionan la paz con la conflictividad en tanto característica de la vida. En este texto se recogen ambas perspectivas de análisis. En el primer caso, se consideran clásicos los estudios de Galtung (2003) acerca de la relación violencia-paz; ellos son fundamentales en la identificación de los supuestos estructurales y culturales básicos para trabajar en función de una paz positiva. La segunda perspectiva tiene que ver con las búsquedas ontológicas y epistémicas que convocan a pensar la paz desde la paz, lo cual demanda aprender a identificar, convivir y regular de manera constante los conflictos. Desde esta óptica la paz imperfecta surge como una apuesta teórica enmarcada en una apertura metodológica concebida en términos transculturales y transdisciplinares que permite "la ampliación del campo de estudio a todos aquellos elementos que pueden considerarse relacionados con un concepto amplio de paz" (Muñoz, 2004, p.38).

Vale la pena advertir que el presente artículo se deriva de la revisión teórica realizada por el autor como parte del trabajo realizado en el marco del Doctorado Paz, Conflictos y Democracia de la Universidad de Granada (España); el trabajo ha contado con la tutoría invaluable de la doctora Carmen Egea Giménez, profesora de la misma Universidad.

\section{Las investigaciones de la paz como tópico de la posguerra}

Un obstáculo serio en las investigaciones en la materia tiene que ver con el hecho de que al estudiar la paz aparece siempre la guerra, de la actuación violenta como forma de definir y enfrentar los conflictos: En ese 
sentido, Martínez advierte, que "los primeros estadios de la investigación de la paz eran más estudios polemológicos, estudios de la guerra, violentología o conflictología que propiamente estudios para la paz" (Martínez, 2004, p.2).

Es después de la segunda guerra mundial cuando la paz parece como temática susceptible de ser abordada científicamente. En la versión de Martínez, Comins y Paris (2009), estos estudios se constituyen como disciplina científica a mediados del siglo XX; su origen se considera como una reacción ante la primera y la segunda guerras mundiales. De acuerdo con estos autores, las dos grandes confrontaciones bélicas de la primera mitad del siglo XX "produjeron un fuerte impacto emocional e intelectual en grupos académicos de muy distinta índole, que tomaron conciencia de la necesidad de reaccionar con los recursos intelectuales a su alcance frente a tal barbarie" (Martínez, et al, 2009, p.4).

En la misma línea de trabajo, el profesor Jiménez Bautista de la Universidad de Granada, identifica tres fases en la investigación para la paz: la fase fundacional; la fase de expansión- especializaciónfragmentación y la fase de hibridación o amalgama (Jiménez, 2009). Con relación a la primera fase, rastrea algunos trabajos hechos durante el siglo $\mathrm{XIX}$, luego define la segunda fase como de exploraciones de la paz a partir de los fenómenos acaecidos en las dos grandes guerras del siglo $\mathrm{XX}$ :

Durante el siglo XIX, cuando los conflictos bélicos parecen conformar la realidad social, el concepto de paz comienza a emerger pero sin una articulación teórica. Es con la Primera y Segunda Guerra Mundial cuando se comienza a elaborar una teoría de la paz. Fueron necesarios el progreso de las ciencias sociales $y$ humanas a lo largo de los siglos XIX y XX y el impacto de las dos guerras mundiales para que se pensara el problema desde una reflexión teórica (Jiménez, 2009, p.148).

La periodización realizada por distintos autores ayuda a identificar tres etapas en los estudios o investigaciones para la paz, de la siguiente manera:

Tres etapas en los estudios o investigaciones para la paz

\begin{tabular}{|c|l|}
\hline ETAPA & \multicolumn{1}{|c|}{ CARACTERÍSTICAS } \\
\hline \multirow{3}{*}{$1930-1959$} & Enfoque violentólogo \\
& Estudio cientifico de la guerra \\
& Concepción de la paz como ausencia de \\
& violencia directa. Paz negativa \\
& Surgimiento de la polemología \\
& Estudio de la guerra (guerra entre Estados) \\
\hline \multirow{3}{*}{$1959-1990$} & Creación del Instituto para lạ Paz de Oslo \\
& Surge el concepto de paz positiva \\
& Énfasis en la justicia y el desarrollo \\
& (satisfacción de necesidades de seguridad, \\
& bienestar, libertad e identidad) \\
\hline \multirow{5}{*}{$1990-$ actualidad } & Se desarrolla el concepto de violencia cultural \\
& Se introduce el concepto de cultura de paz \\
& Énfasis en la educación y la comunicación para \\
& la paz \\
& La paz toma una posición central \\
& Nuevas búsquedas epistémicas y metodológicas. \\
& Paz imperfecta. \\
\hline
\end{tabular}

Fuente: López (2011). Reflexiones sobre las desigualdades en el contexto de los estudios de paz. Revista Paz y Conflictos. Granada: España. 
Kenneth Boulding (1910-1993), aparece en el primer período como el más destacado teórico que aplicó métodos de las ciencias sociales para la generación de logros pacifistas, mediante el uso de técnicas para la resolución de conflictos. Sobre la actuación de Boulding y sus contribuciones, el profesor Juan Carlos Martínez Coll señala:

\begin{abstract}
Junto a su amigo el psicólogo-matemático Anatol Rapoport funda el Center for Research in Conflict Resolution, organización editora del prestigioso Journal of Conflict Resolution, y la Internacional Peace Research Association. La obsesión por la paz originada en la primera Guerra Mundial se refleja en múltiples escritos (Boulding 1945, 1962, 1963, 1993). En los tiempos de la guerra de Vietnam se convierte en un destacado líder del movimiento pacifista, pero su actitud va mucho más lejos que la de un pacifismo reivindicativo elemental. Hay ya, afirma, suficiente «demanda» de paz; lo que se necesita es «oferta». Se trata de ofrecer soluciones alternativas, de elaborar métodos para la resolución de conflictos (Martínez, 2007, párr.15).
\end{abstract}

En sus trabajos finales, Boulding se acerca a una visión optimista de la paz al considerar que ella crece como un proceso casi inconsciente de aprendizaje, lo cual se puede apreciar en la expansión de los territorios con paz estable en los últimos 150 años. Esta interpretación, desde la cual se asume la conflictividad como inherente a la acción humana que en buena medida se resuelve positivamente para las partes, se aproxima a las corrientes más actuales en la materia.

En la actualidad, algunos investigadores notables como Hobsbawm (2007) realizan trabajos alrededor de las expectativas de la guerra y la paz para el siglo XXI. Se trata de análisis históricos y políticos del nuevo orden mundial creado a partir de la estrategia de guerra preventiva de la era Bush. Los trabajos de Hobsbawm son una fuente rica de ideas acerca de los efectos de la globalización sobre las desigualdades del mundo, sobre la crisis del Estado-Nación, el armamentismo generalizado, los conflictos ambientales y las nuevas tensiones generadas por las migraciones y la xenofobia.

El surgimiento de perspectivas más complejas en los estudios conduce a considerar la paz (las paces) no sólo como ausencia de violencia directa o de cesación de hostilidades. Investigadores como Lederach y Galtung, introducen interpretaciones novedosas que permiten ampliar el concepto más allá de las relaciones entre grupos o Estados, involucrando los análisis acerca de la violencia estructural. Desde estas visiones, se considera que no se podría hablar de paz mientras haya relaciones caracterizadas por el dominio y la desigualdad. Ya no se trata exclusivamente de la ausencia de manifestaciones o acciones de violencia directa -la paz como ausencia de algo-, se trata también de avanzar hacia la comprensión de la paz como las condiciones o circunstancias deseadas para su realización acercando de esta manera la paz a la justicia (López, 2011)

En el prólogo del libro Paz por medios pacíficos. Paz y conflicto, desarrollo y civilización (Galtung, 2003), el prologuista Juan Gutiérrez, al referirse a este tránsito de las investigaciones sobre violencia, a partir de la década del sesenta, destaca cómo Galtung y Adam Curle, dieron un giro a la investigación por la paz al reconocer que ella tiene dos lados, uno negativo y otro positivo: "Johan Galtung llama paz positiva al despliegue 
de la vida y paz negativa a la superación de las tres formas de violencia, directa, estructural y cultural" (Galtung, 2003, p.11). La paz positiva hace énfasis en la justicia y el desarrollo, en la satisfacción de necesidades de seguridad, bienestar, libertad e identidad; por tanto, la paz deja de ser la ausencia de guerra para convertirse en un proceso que compromete múltiples visiones y perspectivas teóricas y metodológicas.

Son muy valiosas las contribuciones de Galtung a los estudios sobre la violencia, los conflictos y la paz. La violencia la define como todo lo que obstaculiza la realización completa del potencial humano, tanto física como mentalmente. Al avanzar en la definición diferencia tres tipos: la violencia directa, la violencia estructural y la violencia cultural:

\begin{abstract}
La violencia directa puede dividirse en verbal y física, y en violencia que daña cuerpo y mente o espíritu (...) La violencia estructural se divide en política, represiva y económica, de explotación; respaldada por la penetración, segmentación, fragmentación y marginación estructurales (...). La violencia cultural se divide por su contenido (religión, derecho e ideología, lenguaje, artes, ciencias formales/empíricas, cosmología -cultura profunda-) y por sus transmisores (escuelas, universidades, medios de comunicación) (Galtung, 2003, p.57).
\end{abstract}

Para la transformación de la violencia cultural, Jiménez (2009) propone la paz neutra: la paz actuaría "mediante el diálogo, coherencia de fines y medios, empatía, como el catalizador de las manifestaciones de violencia cultural operantes en las instituciones (...)" (p.159).

Al lado de la escuela de Oslo, se destacan los aportes de Lederach sobre la resolución de conflictos y mediación a partir de un enfoque de transformación de los mismos. Los trabajos de Lederach parten de una amplia experiencia de campo como mediador y negociador en diferentes lugares del mundo: Somalia, Irlanda del Norte, Nicaragua, Colombia y Nepal. Su experiencia en estos procesos lo ha hecho merecedor de un gran reconocimiento en temas de reconciliación desde una perspectiva cristiana. A ellos se deben agregar Rapoport y las investigaciones acerca de las teorías de juegos, integrando las matemáticas, la psicología y la sociología entre otras áreas del conocimiento.

\title{
Nuevos horizontes investigativos para la paz
}

Desde diferentes orillas se producen críticas enconadas al pacifismo, algunas de ellas provienen de representantes notables de la filosofía política contemporánea como Negri (2007) quién considera el pacifismo como "un sentimiento noble pero vacuo", "[y que] la paz puede ser un instrumento de dominio y explotación" [por lo tanto] "hay momentos en que la guerra y la resistencia son necesarias para ser libres y vivir en paz" (Negri, 2007, p.41). En general, las nuevas perspectivas ofrecidas por los estudios para la paz tienen como común denominador un giro epistemológico que consiste en pensar la paz desde la paz y no desde la violencia.

El giro epistemológico plantea la posibilidad de construir una teoría general de los conflictos que, en tanto característica inherente a los seres humanos, no derivan siempre en violencia, por el contrario la mayor parte de ellos desembocan en soluciones pacifistas que es necesario reconocer y potenciar (López, 2011). 
En el texto Endorfinas versus Testosterona: un reconocimiento crítico a Johan Galtung, Muñoz (2004) ayuda a exponer las diferencias en los enfoques de los investigadores que piensan la paz desde la violencia y quienes piensan la paz desde la paz. Los primeros investigadores centran sus mayores esfuerzos intelectuales y teóricos en el estudio de la violencia: La violencia crece mientras la paz se empequeñece- advierte el autor. Las siguientes referencias esclarecen estas diferencias sustanciales:

c) El potente concepto de violencia estructural en caso de ser sobredimensionado puede convertirse en estructuralista. Esto podría permitir, hasta cierto punto, que desaparecieran de la escena los actores o sujetos de la violencia, sus motivaciones y sus causas en este sentido la propuesta de la "racionalidad agónica", de nuestro colega José Manuel Martín Morillas, es muy sugerente

d) Su concepción del conflicto es limitada en cuanto a que es solamente la antesala de la violencia con lo que se pierde perspectiva dialéctica, global y holística. La única alternativa por tanto es esperar como "bomberos de la paz" para intentar que no prenda la violencia. Una perspectiva dialéctica (abierta) nos permitiría relacionar los conflictos con intereses, objetivos, sentimientos y emociones, con actores, tiempos y espacios, las mediaciones donde se "cuecen" y las propuestas de regulación alternativa (Muñoz, 2004, párr.10).

Desde esta óptica la mayor preocupación no está centrada en el reconocimiento y estudio de las violencias como condición para la paz, el avance radica en la comprensión de los conflictos como una característica de los seres humanos que en su mayor parte se han regulado de manera favorable para las partes; ellos son el eje de la base teórica que debe ser desarrollada para explicar tanto la paz como la violencia (Muñoz, 2004).

El estudio de los conflictos implica reconocer la complejidad en tanto conexiones profundas de los fenómenos; el reconocimiento de la imperfección invita a considerar distintas soluciones favorables para las partes en el marco de una conflictividad que no se resuelve de manera completa o absoluta. El hecho de plantear un concepto amplio de paz basada en las soluciones noviolentas que se producen permanentemente como respuesta a los múltiples conflictos, lleva a pensar en la posibilidad de una paz imperfecta revelada como una paz dinámica y perennemente inconclusa. $^{2}$

Esta capacidad para actuar, mediar y resolver pacíficamente los conflictos, señala una ruta ontológica optimista que contrasta con la desesperanza, la incapacidad o el nihilismo enquistado en buena parte de las culturas de occidente, probablemente estimulado por el hecho de que las manifestaciones violentas se hacen más visibles y susceptibles de análisis e intervención. La regulación pacífica, compleja e imperfecta de los conflictos ofrece un marco para la comprensión y actuación ante la vida y la sociedad como parte de la construcción de la paz positiva y la sociedad sustentable.

Buena parte de los marcos interpretativos utilizados por quienes están explorando las posibilidades de reconocer las múltiples expresiones y posibilidades de la paz imperfecta coinciden con los empleados por algunas corrientes del pensamiento ambiental, lo que sigue en el presente 
documento es un intento por señalar esas coincidencias.

\section{La perspectiva ambiental del desarrollo desde el giro epistémico} propuesto en la paz imperfecta

Al enlazar la búsqueda de la paz con la justicia, el bienestar y la generación de una relación no instrumental con la naturaleza, se entrelazan conceptos (¿valores?) como la paz y el desarrollo sustentable. Ambos demandan una racionalidad alterna capaz de transformar los términos actuales sobre los cuales se definen asuntos centrales del pensamiento, la ética y la acción de los sujetos. Las relaciones posibles entre paz y desarrollo sustentable configuran un escenario sobre el cual descansan múltiples alternativas para enfrentar, mitigar o resolver las tensiones que hacen parte de una conflictividad no resuelta.

Como ha sido presentado en trabajos anteriores (particularmente en las conclusiones del ensayo acerca de las experiencias de paz en conflictos fronterizos por el agua. López 2009), en las tres últimas décadas se ha discutido ampliamente en los escenarios académicos y políticos acerca de las implicaciones que para el desarrollo imperante supone la separación entre el entorno natural y el entorno construido. El desarrollo, entendido por las corrientes hegemónicas como proceso de acumulación y crecimiento económico (sustentado en lo que se ha llamado pensamiento único), genera una conflictividad que es causa y efecto de desigualdades (nuevas y viejas) y es, al mismo tiempo, la base de diversas formas de violencia y de cuestionamientos al modelo de sociedad y cultura imperantes. Puede afirmarse que la relación entre la cultura y la naturaleza es altamente conflictiva en razón a las ideologías del desarrollo que asumen la naturaleza como una gran despensa de bienes al servicio de la satisfacción de las inagotables necesidades humanas. Suele decirse que la actual crisis ambiental, al mismo tiempo global y local, interpela de manera profunda los términos actuales del desarrollo y su sustento en una racionalidad cuestionada.

Es probable que la crisis ambiental del desarrollo obligue a reconsiderar la conflictividad como una noción estrictamente antropocéntrica y en su lugar se proponga una conflictividad biocéntrica de manera que el conflicto no sólo se entienda como las divergencias de criterios, intereses, emociones y sentimientos entre los seres humanos, para darle paso a una conflictividad que involucre todas las formas de vida y el conjunto natural; de ahí que sea dable hablar de una cultura de paz como un cultivo de las relaciones con los otros y la naturaleza (Martínez 2000).

Las transformaciones ambientales provocadas por el modelo de desarrollo basado en el crecimiento económico sin límites, y los daños a los ecosistemas derivados de la situación de pobreza y marginalidad en la que se encuentra gran parte de los habitantes del planeta -ligados a una visión reduccionista del desarrollo que ubica a la naturaleza en la condición de recurso económico al mismo tiempo que impulsa una estrategia de crecimiento insostenible a escala global- alcanzan una dimensión insospechada. Como lo ha señalado Kliksberg (2004), los impactos ambientales que profundizan las desigualdades son inmensos:

- En 1950, el 30\% de la Tierra estaba cubierta por bosques: hoy lo está sólo el 20\%. Cada año se pierde un uno por ciento del total de bosques, sobre todo de bosque tropical

- El 34\% de las especies amenazadas son peces, por la pesca excesiva y sin control que realizan algunos países. La desertificación afecta al 35\% de la superficie del planeta 
- En el último cuarto del siglo XX se perdió el $18 \%$ de las tierras cultivables. El $15 \%$ de la población vive en países ricos y consume el $56 \%$ de los recursos mundiales

- Como los bienes y servicios ecosistémicos son en principio libres o están muy subvalorados en el mercado, los agentes económicos tienden sistemáticamente a su sobreexplotación (Klikgsberg, 2004, en Villegas y López, 2006, pp. 56-57).

A lo anterior se agregan daños a escalas mayores como el incremento de la temperatura en el planeta, la disminución de la capa de ozono y el deterioro generalizado de valiosos ecosistemas que han provocado una crisis ambiental con implicaciones globales; la crisis ha puesto en evidencia el carácter agónico de la racionalidad que soporta las ideologías del progreso. Como lo ha sentenciado Martínez (2000) el problema real del desarrollo no es su eventual fracaso sino su éxito. Desde la perspectiva teórica de la paz imperfecta se advierte que la relación naturaleza-cultura demanda un giro epistemológico que permita generar relacionamientos sustentables basados en los aprendizajes y experiencias de diversas comunidades. Algunos de los ejes del giro epistemológico son los siguientes:
(...) Frente a la objetividad, intersubjetividad e interpelación humana. Sustituir la perspectiva del observador distante por la de participante. El conocimiento deja de ser una relación entre sujeto y objeto para convertirse en una relación entre sujetos. Referir se entiende como una de las acciones humanas y nuestro campo de estudio es lo que nos hacemos unos a otros. No ser neutrales respecto de valores. Pasar al paradigma de la comunicación (...) (Martínez, 2000, p.90).

Buena parte de la literatura que hace parte del pensamiento ambiental sostiene que las condiciones actuales de producción, distribución y consumo ha cruzado los umbrales de irreversibilidad, momento a partir del cual se generan los daños ambientales; esta tendencia provoca, a su vez, al aumento en la complejidad, la frecuencia y la magnitud de los impactos ambientales, los cambios generalizados en las condiciones físicas del planeta, con efectos tales como la globalización de los problemas derivados de la escasez de las materias primas y la generación de luchas políticas por los recursos estratégicos. En clave pacifista y siguiendo a Muñoz (2004b), podría afirmarse que la racionalidad en su avance desata fenómenos paradójicos: paz y violencia, vida y muerte.

Una valoración general de las problemáticas ambientales invita a afirmar que el desarrollo económico y social insostenibles está lejos de ser reemplazado por visiones y prácticas sustentables, las soluciones técnicas -tecnologías limpias y mercados verdes- no han dado los resultados anunciados en las escalas esperadas; los procesos biológicos necesitan un tiempo considerablemente mayor que el que les permite la presión humana sobre los ecosistemas; los sistemas sociales tienden a mantenerse inamovibles a pesar de los indicadores de crisis; los conflictos ambientales son focos de luchas políticas interestatales y de nuevas desigualdades y, en consecuencia, es evidente que, el progreso continuo ha aumentado los problemas ambientales generando una conflictividad que necesita ser resuelta mediante transformaciones culturales (Villegas y López, 2006).

Los desafíos para el desarrollo sostenible resultan ser los mismos que enfrenta la paz imperfecta. En el terreno epistemológico ambas perspectivas se apoyan en la gestión de la complejidad, la comprensión de los sistemas complejos, la superación de la fragmentación del 
conocimiento -rompiendo, entre otros, el falso dualismo culturanaturaleza-, el reconocimiento de la transdisciplinariedad, el empleo de múltiples metodologías y la transculturalidad en los distintos abordajes (López, 2009).

En las tres últimas décadas, la Organización de las Naciones Unidas ha convocado a los gobernantes de todos los países a una multiplicidad de eventos buscando avanzar en la definición y puesta en marcha de acuerdos internacionales y políticas nacionales bajo los principios de sostenibilidad ambiental del desarrollo ${ }^{3}$, cada uno de estos foros ha permitido avanzar sobre los conceptos y los alcances de la perspectiva ambiental en conexión con valores pacifistas. Así por ejemplo, la cumbre de Río (1992), planteó que el derecho al desarrollo debe ejercerse de forma que responda equitativamente a las necesidades ambientales y de desarrollo de generaciones presentes y futuras buscando superar las desigualdades imperantes, en una visión que puede ser evaluada como una orientación de las acciones del desarrollo hacia la búsqueda de lo que Galtung ha definido como paz positiva estructural. En el caso de la cumbre de Johannesburgo (2002) la agenda contempló la revisión de la situación ambiental en el marco de la pobreza; a pesar de los reconocimientos colectivos sobre la urgencia de construir una racionalidad ambiental distinta quedó en evidencia la profundización de tres grandes crisis que en su interrelación configuran la problemática ambiental del desarrollo: la desigualdad estructural, expresada en la pobreza, la marginalidad y la concentración de la riqueza y el ingreso que padecen poblaciones extendidas por vastas zonas de la tierra; la crisis de existencia reflejada en el sometimiento humano a las condiciones que impone la sociedad de consumo, las múltiples formas de violencia y la banalización de la vida, y la crisis ecológica dada la devastación de los ecosistemas y la presión ejercida sobre la biósfera.

Lo que actualmente se conoce como desarrollo sostenible ha sido el resultado de numerosos estudios, encuentros locales e internacionales y movilizaciones sociales y políticas. Los encuentros oficiales y alternos en las cumbres de Rio y Johannesburgo (sólo por señalar dos situaciones emblemáticas) contribuyeron a que se difundieran distintas corrientes de pensamiento y diversas orientaciones que acompañan las acciones de ambientalistas y pacifistas. Como se sabe, buena parte de las extensas deliberaciones de las conferencias mundiales quedan en entredicho al momento de suscribir y poner en operación los grandes acuerdos por parte de los líderes mundiales comprometidos con un modelo hegemónico de desarrollo, lo cual, a su vez, se traduce en bajos alcances prácticos de las propuestas que logran consensos.

Luego de la cumbre de Johannesburgo, se han realizado múltiples eventos dirigidos a evaluar los resultados de los encuentros mundiales anteriores; la agenda central ha estado copada por las discusiones ligadas a los efectos del cambio climático. Al tiempo que algunos países suscriben pactos de reducción de emisiones contaminantes, otros esgrimen el derecho a seguir una senda del desarrollo centrada en el crecimiento económico. Los eventos han puesto en evidencia la presencia de una doble racionalidad de los gobernantes ante los retos de la paz y el desarrollo. En lenguaje de paz imperfecta puede decirse qué, no obstante la reticencia de los países más poderosos, las cumbres han sido útiles para poner en evidencia los incontables pequeños logros locales que sostienen la esperanza de una sociedad pacífica y sustentable.

\section{Consideración final}

Las reflexiones teóricas y las acciones ejemplares para la paz involucran un amplio y destacado grupo de personalidades cuyas obras han sido fundamentales en la construcción de una visión pacifista del mundo y de 
la vida, algunas de ellas han sido reconocidas como influencias directas en las luchas y reivindicaciones de los ambientalistas. La lista incluye nombres notables como Henry D. Thoreau, León Tolstoi, M. C Gandhi, Albert Einstein, Virginia Woolf, Hanna Arendt, Martin Luther King y Bertrand Russell, entre otros. El pensamiento y las acciones políticas de estas personalidades siguen influyendo en las reflexiones y propuestas alternas actuales.

Se consideran centrales los aportes de Thoreau en el campo de la resistencia civil, su rechazo a la esclavitud y su defensa del derecho de las personas a la desobediencia. En el caso de Tolstoi son notables la búsqueda del auto perfeccionamiento moral, la desobediencia a los mandatos del Estado y el rechazo a la fuerza como método para resolver los conflictos. El legado pacifista de Einstein se reconoce especialmente en su antimilitarismo radical (en particular la oposición al servicio militar obligatorio antes del triunfo Nazi y las convocatorias posteriores al desarme); en la misma línea, se puede ubicar la lucha contra el nazismo de Virginia Woolf (la visión de las mujeres sobre la guerra y el cómo evitarla), y la lucha por el reconocimiento de los derechos civiles en los Estados Unidos por parte de Martín Luther King (Prat, ed. 2004).

Desde el lado del ambientalismo probablemente resulte más comprometedor por su extensión y heterogeneidad teóricas realizar una selección de las corrientes del pensamiento y de las personalidades emblemáticas que se han ocupado de estudiar y actuar sobre las problemáticas del desarrollo con implicaciones sobre el pensamiento y la acción de los pacifistas. Una influencia decisiva en los ámbitos académicos más cercanos se debe reconocer en los trabajos del Maestro Augusto Ángel Maya ${ }^{4}$, ellos resultan indispensables en cualquier intento de organización del pensamiento ambiental y su influencia sobre otras corrientes del pensamiento; en todo caso, para un abordaje académico de esta naturaleza, se dispone de una amplísima bibliografía con líneas editoriales alimentadas permanentemente por trabajos que abarcan desde el capitalismo verde, cuyo énfasis está en la generación de alternativas sostenibles para el modelo económico imperante, hasta concepciones quizás influidas por la ecología profunda en cuyo centro está la identificación de las fallas de la cultura y la convocatoria a realizar un cambio de piel cultural.

A pesar de las críticas al pacifismo por parte de quienes lo consideran un sentimiento noble pero vacuo -y de los juicios radicales a los pacifistas por parte de quienes pregonan las guerras y las resistencias violentas como prerrequisitos indispensables para alcanzar la paz-, las investigaciones basadas en valores pacifistas han logrado un lugar cada vez más visible en las ciencias humanas y sociales. Las más recientes perspectivas para la paz, especialmente las que se centran en el examen de la paz desde la paz con fundamento en el análisis de los conflictos, se aproximan a las epistemes que fundamentan el pensamiento de los ambientalistas basado en la potencialidad de la razón sustentable, en ambos casos se pone en entredicho la experiencia del desarrollo fundamentada en individuos y sociedades codiciosas, violentas, insaciables e insostenibles. 


\section{REFERENCIAS BIBLIOGRÁFICAS}

- Alvear, J. (2008). La paz neoliberal: el postulado de la razón instrumental sobre la razón dialógica. Versión electrónica, Criterio Jurídico 8, 2. Santiago de Cali.

- Galtung, J. (1998). Tras la violencia, 3 R.: reconstrucción, reconciliación, resolución. Afrontando los efectos visibles e invisibles de la guerra y la violencia. Bilbao: Gernika Gogoratuz.

- Galtung, J. (2003). Paz por medios pacíficos. Paz y conflicto, desarrollo y civilización. Bilbao: Gernika Gogoratus.

- Hobsbawm, E. (2007). Guerra y paz en el siglo XXI. Barcelona: Editorial Crítica.

- Jiménez, F. (2009). Hacia un paradigma pacífico: la paz neutra. Versión electrónica, Convergencia Revista de Ciencias Sociales, IA. México: Universidad Autónoma del Estado de México.

- Kliksberg, B. (2004). ¿Cómo reformar el Estado para enfrentar los nuevos desafíos sociales? Revista Administración \& Desarrollo, 41, 10 - 41. Facultad de Investigaciones -ESAP.

- López, M. H. (2009) Experiencias de paz en conflictos fronterizos por el agua. Revista Luna azul. 28, 96-102. Manizales: Universidad de Caldas. En: http://200.21.104.25/lunazul/downloads/Lunazul28_10.pdf

- López, M.H. (2011) Reflexiones sobre las desigualdades en el contexto de los estudios de paz. Revista Paz y Conflictos, 4, 115. España: Universidad de Granada. En: http://www.ugr.es/ revpaz/tesinas/rpc_n4_2011_dea2.pdf

- Martínez, J. C. (2007). Kenneth E. Boulding, economista y pacifista. Textos de economía, paz y seguridad, 1, 1. Recuperado el 23 de junio de 2010 En: http://www.eumed.net/rev/tepys/01/jcmc-1.htm

- Martínez, V. (2000, septiembre - diciembre). Saber hacer las paces. Epistemologías de los estudios para la paz. Convergencia, Revista de Ciencias Sociales, 7, 23. México: Universidad Autónoma del Estado de México.

- Martínez, V. (2004). Teorías de la guerra en el contexto político de comienzos del siglo XXI. Recuperado el 15 de abril de 2010 En: http://www.ugr.es/ fmunoz/documentos/Teor\%C3\%ADas\%20de \%20la\%20guerra\%20Siglo\%20XXI\%20no\%20endnote.pdf

- Martínez, V.; Comins, I. y Paris, A. (2009). La nueva agenda de la filosofía para el siglo XXI: los estudios para la paz. Convergencia [en línea]. Recuperado el 20 de abril de 2010 En: http://redalyc.uaemex.mx/src/inicio/ArtPdfRed.jsp?iCve=1051224 4005.

- Maya, A. (1995). La fragilidad ambiental de la cultura. Bogotá: Universidad Nacional.

- Muñoz, F. (2004). La paz. En B. Molina y F. Muñoz (Eds.), Manual de paz y conflictos. Granada: Universidad de Granada.

- Muñoz, F. (2004). Qué son los conflictos. En: Molina, B. y Muñoz, F. Manual de Paz y Conflictos. Granada: Universidad de Granada.

- Muñoz, F. (2004b). Endorfinas versus Testosterona. Un reconocimiento crítico a Johan Galtung. Versión para estudiantes. En, H:IDocumentos web-desigualdadesldocumentos pazlEndorfina V.s. testosterona. Un reconocimiento crítico a Johan Galtung_archivoslgaltungcrit.htm

- Negri, A. (2007). Goodbye MR. socialism. La crisis de la izquierda y los nuevos movimientos revolucionarios. Barcelona: Ediciones Paidós.

- Prat, E. (2004). Pensamiento pacifista. Barcelona: Icaria.

- Ricó-Bernabé, R. (2004). El mantenimiento de la paz ante los retos de las nuevas guerras. Barcelona: Icaria Editorial. 
- Villegas, G. y López, M. H. (2006). La gestión ambiental de las PYMES en Manizales. El interés ambiental y el consumo socialmente responsable del empresario. Manizales, Colombia: Universidad de Caldas.

1. Profesor Universidad de Caldas. Departamento de Economía y Administración

2. Una definición de paz imperfecta se encuentra en los documentos del curso de Fundamentos de los estudios para la paz y los Derechos Humanos: el concepto de paz imperfecta se usa para definir "aquellos espacios e instancias en las que se pueden detectar acciones que crean paz, a pesar de que estén en contextos en los que existen los conflictos y la violencia". Documentos de clase, Muñoz y Molina (2009).

3. Existe una diferencia entre desarrollo sostenible y sustentable en función de los alcances del giro epistemológico. Para efectos de este texto, se hace referencia al desarrollo sostenible tal y como se acuñó en la Conferencia de Estocolmo realizada en 1972.

4. Algunos de sus trabajos se proponen desarrollar un método ambiental de interpretación de la historia. En ellos se aleja de los ecologismos convencionales para adentrarse en la necesidad de formar una nueva sociedad, planteándose, de esta manera, retos similares a los expuestos recientemente por los pacifistas: "El problema ambiental [advierte] está, por lo general, mal planteado en la arena del debate teórico y de la acción práctica. No es un problema que atañe solamente a los ecosistemas naturales o que se pueda solucionar simplemente con medidas tecnológicas. Requiere la formación de una nueva sociedad" (Maya, 1995, p 11). 